\title{
Factors influencing cartilage tympanic perforation repair in children.
}

\author{
François SIMON ${ }^{1}$, Briac Thierry ${ }^{1}$, Tioka Rabeony ${ }^{1}$, Florian Verrier ${ }^{1}$, Caroline Elie ${ }^{1}$, \\ Natalie Loundon ${ }^{1}$, Nicolas Leboulanger ${ }^{1}$, Vincent Couloigner ${ }^{1}$, Erea-Noël Garabédian ${ }^{1}$, and \\ Françoise Denoyelle ${ }^{1}$ \\ ${ }^{1}$ Necker-Enfants Malades Hospitals
}

March 18, 2021

\begin{abstract}
Objectives: The aim of the study was to identify factors that could influence the repair of eardrum perforation using cartilage graft (or cartilage tympanoplasty) in children. Methods: A cohort of children operated on between January 1998 and December 2012 was reviewed. We have studied the repair rate of the eardrum (anatomical result) and the hearing level with audiometric tests (functional result) at 1 year and 3 years after surgery. These results were correlated with size or location of the perforation, status of the contralateral ear, gender, allergies, cleft palate, craniofacial anomalies, expertise of the surgeon (junior, senior) and perioperative observations (mucosa, glue, etc.). Results: 1240 tympanoplasties were selected from the database, of which 139 ears (127 patients) could be analysed (perforation without concurrent disease, authorisation from patients obtained and sufficient information reported). Mean age at surgery was 9.6 years \pm 2.6 (range 4-16). At one year, 129/139 (93\%) tympanic membranes were closed and 112/139 (81\%) were satisfactory (no residual perforation, nor retraction, cholesteatoma, myringitis or OME). Air-bone gap was $<20 \mathrm{~dB}$ in 102/127 ears (80\%). At 3 years, the eardrum was closed in 64/66 (97\%) ears (reperforation in one case) and $82 \%$ were satisfactory. Myringitis occurred in $5 \%$ and $9 \%$ of cases at one- and three-year follow-up. Surgery before the age of 8 years was the only risk factor of a non-satisfactory result at one-year follow-up $(\mathrm{p}=0.024)$. Conclusions: Long-term results were satisfactory; the only risk factor was surgery before eight years of age. In the child, long-term yearly follow-up is necessary after tympanic perforation.
\end{abstract}

Factors influencing cartilage tympanic perforation repair in children.

\begin{abstract}
Objectives: The aim of the study was to identify factors that could influence the repair of eardrum perforation using cartilage graft (or cartilage tympanoplasty) in children.

Methods: A cohort of children operated on between January 1998 and December 2012 was reviewed. We have studied the repair rate of the eardrum (anatomical result) and the hearing level with audiometric tests (functional result) at 1 year and 3 years after surgery. These results were correlated with size or location of the perforation, status of the contralateral ear, gender, allergies, cleft palate, craniofacial anomalies, expertise of the surgeon (junior, senior) and perioperative observations (mucosa, glue, etc.).

Results: 1240 tympanoplasties were selected from the database, of which 139 ears (127 patients) could be analysed (perforation without concurrent disease, authorisation from patients obtained and sufficient information reported). Mean age at surgery was 9.6 years \pm 2.6 (range 4-16). At one year, 129/139 (93\%) tympanic membranes were closed and 112/139 (81\%) were satisfactory (no residual perforation, nor retraction, cholesteatoma, myringitis or OME). Air-bone gap was $<20 \mathrm{~dB}$ in 102/127 ears (80\%). At 3 years, the eardrum was closed in 64/66 (97\%) ears (reperforation in one case) and $82 \%$ were satisfactory. Myringitis
\end{abstract}


occurred in $5 \%$ and $9 \%$ of cases at one- and three-year follow-up. Surgery before the age of 8 years was the only risk factor of a non-satisfactory result at one-year follow-up $(\mathrm{p}=0.024)$.

Conclusions: Long-term results were satisfactory; the only risk factor was surgery before eight years of age. In the child, long-term yearly follow-up is necessary after tympanic perforation.

Keywords: tympanoplasty; perforation; cartilage; child, pediatric, paediatric

\section{Key points:}

- Tympanoplasty for tympanic perforation in children requires prolonged yearly follow-up

- Tympanic perforation closure definition of success should not only include anatomical closure but also hearing results and absence of complications such as myringitis

- Tympanic closure before the age of eight was the only factor associated with a lower success rate at one-year follow-up

- Results at one-year follow-up may not be stable in the child, as one in ten satisfactory eardrums at one-year may become unsatisfactory at three-year follow-up

- Myringitis is the main complication at one and three-year follow-up, occurring in 5-8\% of cases

\section{Introduction:}

In children with tympanic membrane perforation, tympanoplasty aims to reconstruct the tympanic membrane to allow swimming and to create a well aerated, healthy, and hearing middle ear. Numerous surgical techniques have been developed and various graft materials used for the closure of tympanic membrane perforations, mainly fascia, fat, cartilage and perichondrium. The increased frequency of upper respiratory tract infections, otitis media, and eustachian tube dysfunction makes children at higher risk of surgical failure after tympanoplasty compared to adults (1).

Cartilage has become the preferential material for tympanic perforation closure in the child, due to its resistance in unfavourable local conditions, such as chronic infection or middle ear depression, which are frequent in the child. These may immediately impede healing or occur after closure and alter long-term results $(2-5)$.

The importance of different factors and especially age at surgery remains debated in the literature (6-12). Also, the tympanic closure rate at 12 months follow-up or less does not seem sufficient to assess the success of the procedure in paediatric patients, due to frequent otitis media with effusion (OME) or post-operative retraction pockets or cholesteatoma in children $(7,9,11,13)$. Long-term success rate should include tympanic closure without OME or post-operative complication and audiological results $(13,14)$.

The aim of the study was to determine the factors that could influence the audiometric and functional results of cartilage tympanoplasty in children with tympanic membrane perforation, in a large cohort of children operated on with the same surgical procedure by paediatric otologists. The secondary objective was to determine the long-term three-year prognosis.

\section{Materials and Methods:}

This retrospective cohort study followed STROBE guidelines. The patient inclusion process is detailed in Figure 1. All consecutive patients, aged $<18$ years old, operated on between January 1998 and December 2012 for tympanic membrane (TM) perforation closure using a cartilage underlay technique were included. Patients had to have a one-year follow-up (including anatomical examination and audiometry workup) and have no history of ipsilateral ear surgery (except for ventilation tubes). Medical files of all perforations were reviewed using FileMaker Pro (Claris, Santa Clara, California, USA) and per, perioperative and surgical data was analysed. Patient age at surgery, sex and comorbidities (allergy, cleft palate, craniofacial anomaly) 
were noted. Also, perforation size, location, surgical technique, expertise of the surgeon (junior surgeon or senior paediatric otologist), status of the contralateral ear and perioperative observations (mucosa inflammation, glue in the middle ear) were noted. The following postoperative information was noted: TM closure, myringitis, otitis media with effusion (OME), TM retraction, cholesteatoma, external auditory canal stenosis. Audiometric tests, air and bone conduction, mean air-bone gap, were reported using the pure-tone average (PTA) referring to the average of hearing threshold levels at 500, 1000, 2000 and 4000Hz. Statistical analysis was made using R software (http://cran.r-project.org/). Data was described as mean \pm standard deviation or median [interquartile range] for quantitative variables and frequencies (\%) for qualitative variables. Comparisons for continuous data were performed through Student test or Wilcoxon rank test and for categorical data through Pearson's Chi-squared test or Fisher's Exact Test. Statistical tests were two-sided and $\mathrm{p}$ values less than 0.05 were considered statistically significant. This is a retrospective study and has been approved by our local Institution Review Board (IRB). The study was registered on the Clinical Trials Database (NCT02296944). In compliance with French law, a letter of no objection was sent to all participants if they were adults or otherwise their parents. The data that support the findings of this study are available on request from the corresponding author. The data are not publicly available due to privacy or ethical restrictions.

\section{Results:}

In this study, 139 ears (127 patients) were included, 57 males and 70 females. Comorbidities were as follows: 16 patients (13\%) had craniofacial malformations (including ten with a velopalatal cleft), 25 patients were diagnosed with allergic rhinitis $(20 \%)$.

Mean age at surgery was 9.6 years \pm 2.6 (Figure 2 ). Perforation was in the right ear in 74 cases $(53 \%$ of ears) and in the left ear in 65 cases (47\%). No clinical symptom was present in 49 cases (35\%). Preoperative auditory evaluation is reported in Table 1 . The size of the perforation was available for 109 ears (78\%): pinpoint size one ear (1\%), up to a quarter of the eardrum's surface in 34 ears (31\%), up to half of the eardrum in 38 ears $(35 \%)$ and more than half of the surface to total in $36(33 \%)$. Location of the perforation was available in 137 ears (99\%): posterior in 24 cases (18\%), inferior in 28 cases (20\%), anterior in 47 cases (34\%), subtotal or central in 36 cases $(26 \%)$ and multiple in two $(2 \%)$.

All procedures were performed with an endaural approach and an underlay tragal cartilage graft. Procedures were performed by a senior paediatric otologist in 57 cases $(47 \%)$ and by a junior otolaryngologist (10 years' experience or less) in 65 cases $(53 \%)$. There was a preoperative external auditory canal stenosis in 17 cases $(12 \%)$. During the procedure, the atrial mucosa was inflammatory (abnormal mucosa or otorrhoea) in 45 ears $(33 \%)$ and glue in the middle ear was found in nine ears $(7 \%)$.

At one-year anatomical assessment follow-up, 129 (93\%) tympanic membranes were closed. Satisfactory anatomical result (closure without OME, retraction, cholesteatoma or myringitis) was observed in 112 ears (81\%). The unsatisfactory results are detailed in Table 2 . Notably, two children had developed a severe retraction pocket with cholesteatoma and nine an evolutive retraction pocket at one-year follow-up. Of those patients, $3 / 11$ had cleft palate, $2 / 11$ had contralateral tympanoplasty and mean age at surgery was 9.2 years.

Audiometry data one year after surgery were available for 127 ears (91\%) and reported in Table 1 . Audiometric results were considered good (residual air conduction [?] $20 \mathrm{~dB}$ ) in 102 ears (80\%). In five ears (4\%) the bone conduction threshold was increased by more than $10 \mathrm{~dB}$. Both anatomical and audiometric success (satisfactory anatomical and audiometric results) was obtained in 84 ears $(66 \%)$.

The otoscopic data at three-years post-surgery were available for 66 ears (47\% of 139 ears). Among the 52 ears which were satisfactory at one year, six $(12 \%)$ became unsatisfactory at three years (including one reperforation, two OME and three cases of myringitis). Of the 14 ears which were unsatisfactory at oneyear follow-up, 10 became satisfactory and four remained unsatisfactory (including one cholesteatoma, one perforation with myringitis and two cases of myringitis). Overall, at three years follow-up, the tympanic 
membrane was closed in 64/66 ears (97\%) and the result was satisfactory in 56/66 ears (85\%). Myringitis was the most frequent cause of poor result (six ears, $9 \%$ ), detail in Table 2 . The median air conduction threshold was $15 \mathrm{~dB}[12.5 ; 22.5]$ with $26 / 32(81 \%)$ of ears considered as having a good functional result (auditory data available for 32 ears, detail in Table 1 ). Overall success at three years (both satisfactory anatomical and audiometric results) was obtained in 24/32 ears (75\%). Air conduction audiometry was significantly better at one-year and three-years follow-up versus pre-operative values (difference at 1 year $-6 \mathrm{~dB}[-10 ; 0] \mathrm{p}<0.001$; difference at 3 years $-2 \mathrm{~dB}[-13 ; 1] \mathrm{p}=0.034)$.

The univariate analysis of risk factors of non-satisfactory TM at one-year follow-up identified only one significant risk factor. Patients operated on under the age of eight years had $31 \%$ of non-satisfactory results at one-year follow-up (versus $14 \%$ at the age of eight or over, $\mathrm{p}=0.024$ ). The other risk factors (size, anterior or subtotal perforation, various associated pathologies, other age groups) were not associated with higher risk of non-satisfactory TM (Table 3 ). When analysing tympanic perforation locations separately (posterior, inferior/central, anterior, subtotal or multiple locations), no statistical difference in outcome at one year was found $(\mathrm{p}=0.20)$.

\section{Discussion:}

The rate of $93 \%$ of TM closure at one year reported in this series corresponds to other large paediatric studies, ranging from 74 to $97 \%(1,5-7,9-12,15-18)$. However, our $81 \%$ TM satisfaction rate at one year, which includes complications linked to Eustachian tube malfunction, seems to us more appropriate and clinically relevant in paediatric patients. Furthermore, when associating satisfactory anatomical and audiometric result, success rate falls to $66 \%$ at one year. It has been shown in previous studies that OME, re-perforation and tympanic retractions are commonplace in the closed TM of the child $(7,9,11,13,18)$. We believe that these complications directly linked to the correct surgical TM closure, should be taken into account to determine if the procedure was a success. As these complications sometimes occur between one and three years after surgery, we believe that the minimum duration of follow-up should be of three year, for the surgery to be considered successful $(9,10)$.

At 3 years, the rate of reperforation was very low (one case out of $66,2 \%$ ) but the rate of myringitis was surprisingly high (9\%) and could be a consequence of the use of cartilage to close the TM. Although no statistical link has been established, it has been described previously in other cartilage tympanoplasty studies $(14,15)$. No specific clinical characteristic was identified in children with myringitis. The rate of myringitis remained important over time (5\% at one year and $9 \%$ at three years). However, this may also be explained by the smaller number of patients at the three-year follow-up (66 vs 139) and that patients with post-operative complications are more likely than asymptomatic patients to return for long-term follow-up after myringoplasty.

The univariate study showed that the size and location of the TM perforation were not risk factors (the success rate for anterior and larger perforations was only of $76 \%$ but was not significantly lower) and found significantly better results when the procedure was performed at eight-years old or older. In the literature, the role of size and location of the perforation has been reported as a factor influencing the results, large and anterior locations being associated with poorer outcomes $(8,9,17)$. The role of age is controversial, as it has not been found to be a significant factor for success in some studies (5-8), while others also consider younger age being a risk factor, setting the cut-off at eight $(9)$, nine $(11,12)$ or ten years of age $(10)$. Another study found that children operated on before the age of eight had a significantly higher rate of re-perforation (19).

Concerning other factors, as in our study, craniofacial comorbidities were not associated with poorer outcomes in three studies $(7,14,19)$, including patients with velopalatal clefts. Too few patients with such comorbidities were included in our cohort to properly address their influence on the rate of successful tympanic membrane perforation closure. Perioperative tympanic cavity inflammation has previously been identified as a risk factor (5) however as in our study, a meta-analysis found statistically non-significant decreased success 
rate (8). Chronic otitis in the contralateral ear has been associated with poorer outcomes in some studies $(5,8,20)$ but, has also as in our series not been associated with statistically significant results (17). This discrepancy might result from the fact that following a previous publication (5), ipsilateral inflammatory mucosa and contralateral OME have been considered relative contraindications of tympanic closure in our team. Consequently, only very few patients with these two factors were included in the present series, biasing the statistical analysis.

Two main limitations can be identified in this study. First the lost-to-follow-up rate which could induce selection bias influencing our success rate, as those patients might have stopped their follow-up due to the absence of symptoms or sought a follow-up elsewhere if complications occurred. Second is the high proportion of children with a history of craniofacial malformations (13\%), and especially velopalatal clefts (8\%), in our cohort. One of the reasons is that our paediatric hospital concentrates a large number of national referral centres for malformative diseases. Those children are more likely to develop ear ventilation problems, OME and retraction after tympanoplasty (14). Additionally, the high percentage of craniofacial malformations in our series and the fact that most children with such anomalies benefit from one consultation per semester or per year throughout their childhood and adolescence in our center, might explain the longer median follow-up duration in our study as compared to most previous publications, thus inducing a follow-up bias

Thus, a minimum of eight years of age at the time of surgery seems to be preferable and long-term follow-up over a few years would be advisable complications may occur in the long-run.

\section{Conclusion:}

In this study, a large cohort of patients was reviewed, with a one-year follow-up and with an identical surgical technique (underlay tragal cartilage). We emphasise that, specifically in the child, success after tympanic perforation surgery should be assessed in the long-run, with a follow-up of at least one year for all patients, and should not only focus on TM closure but also include the general aspect of the TM and audiometric outcome. Our results tend to show that tympanic closure should be best performed at the age of eight or older and that myringitis is the most common long-term complication (5 to 9\%). Eustachian tube malfunction in the younger child seems to increase the risk not only of tympanic closure failure, but also subsequent cholesteatoma, retraction or OME. Due to the high success rate after tympanoplasty for perforation in the child (80-90\%), very large prospective and multicentric studies are necessary to better identify risk factors and the indications of different techniques (cartilage or fascia grafts, underlay, overlay, butterfly, fat plug, etc.).

\section{References:}

1. Yilmaz MS, Guven M, Kayabasoglu G, Varli AF. Comparison of the anatomic and hearing outcomes of cartilage type 1 tympanoplasty in pediatric and adult patients. Eur Arch Otorhinolaryngol. 2015 Mar;272(3):557-62.

2. Vashishth A, Mathur NN, Choudhary SR, Bhardwaj A. Clinical advantages of cartilage palisades over temporalis fascia in type I tympanoplasty. Auris Nasus Larynx. 2014 Oct;41(5):422-7.

3. Jalali MM, Motasaddi M, Kouhi A, Dabiri S, Soleimani R. Comparison of cartilage with temporalis fascia tympanoplasty: A meta-analysis of comparative studies. Laryngoscope. 2017;127(9):2139-48.

4. Kalcioglu MT, Tuysuz O, Yalcin MZ, Karatas E. Does cartilage thickness affect hearing results in real life? Long-term results of cartilage and fascia graft in type 1 tympanoplasty. Clin Otolaryngol. 2019 Sep;44(5):842-6. 
5. Denoyelle F, Roger G, Chauvin P, Garabedian EN. Myringoplasty in children: predictive factors of outcome. Laryngoscope. 1999 Jan;109(1):47-51.

6. Singh GB, Arora R, Garg S, Kumar S, Kumar D. Paediatric tympanoplasty: comparative study between patients aged 5-8 years and those aged over 14 years. J Laryngol Otol. 2016 Jul;130(7):635-9.

7. Sanchez Barrueco A, Lora Pablos D, Villafruela Sanz M, Almodovar Alvarez C. Pediatric myringoplasty: Prognostic factors in surgical outcome and hearing threshold recovery. Acta Otolaryngol. 2015;135(12):12337.

8. Hardman J, Muzaffar J, Nankivell P, Coulson C. Tympanoplasty for Chronic Tympanic Membrane Perforation in Children: Systematic Review and Meta-analysis. Otol Neurotol. 2015 Jun;36(5):796-804.

9. Kumar S, Acharya A, Hadjihannas E, Panagamuwa C, McDermott AL. Pediatric myringoplasty: definition of "success" and factors affecting outcome. Otol Neurotol. 2010 Dec;31(9):1417-20.

10. Takahashi-Tatsumi E, Mishiro Y, Katsura H, Sakaguchi A, Sakagami M. Longitudinal follow-up after pediatric myringoplasty: long-term outcome is defined at 12 months. Otol Neurotol. 2014 Jan;35(1):126-8.

11. Rozendorn N, Wolf M, Yakirevich A, Shapira Y, Carmel E. Myringoplasty in children. Int J Pediatr Otorhinolaryngol. 2016 Nov;90:245-50.

12. Sckolnick JS, Mantle B, Li J, Chi DH. Pediatric myringoplasty: factors that affect success-a retrospective study. Laryngoscope. 2008 Apr;118(4):723-9.

13. Nevoux J, Roger G, Chauvin P, Denoyelle F, Garabedian EN. Cartilage Shield Tympanoplasty in Children: Review of 268 Consecutive Cases. Arch Otolaryngol Head Neck Surg. 2011 Jan 17;137(1):24.

14. Harterink E, Leboulanger N, Kotti S, Garabedian E, Denoyelle F. Results of myringoplasty in children with cleft palate: a patient-matched study. Otol Neurotol. 2014;35:838-43.

15. Kaya I, Benzer M, Gode S, Sahin F, Bilgen C, Kirazli T. Pediatric type 1 cartilage tympanoplasty outcomes: A comparison of short and long term hearing results. Auris Nasus Larynx. 2018 Aug;45(4):7227.

16. James AL. Endoscope or microscope-guided pediatric tympanoplasty? Comparison of grafting technique and outcome. Laryngoscope. 2017;127(11):2659-64.

17. Knapik M, Saliba I. Pediatric myringoplasty: a study of factors affecting outcome. Int J Pediatr Otorhinolaryngol. 2011 Jun;75(6):818-23.

18. Couloigner V, Baculard F, El Bakkouri W, Viala P, Francois M, Narcy P, et al. Inlay butterfly cartilage tympanoplasty in children. Otol Neurotol. 2005;26:247-51.

19. Duval M, Grimmer JF, Meier J, Muntz HR, Park AH. The effect of age on pediatric tympanoplasty outcomes: a comparison of preschool and older children. Int J Pediatr Otorhinolaryngol. 2015 Mar;79(3):336-41.

20. Collins WO, Telischi FF, Balkany TJ, Buchman CA. Pediatric tympanoplasty: effect of contralateral ear status on outcomes. Arch Otolaryngol Head Neck Surg. 2003 Jun;129(6):646-51.

\section{Table 1: Audiometric data}

Characteristics

Number of ears

Good functional result ${ }^{\S}$

Good functional result and satisfactory anatomical result

Bone conduction PTA

Median [Q1; Q3]

Range 
Air conduction PTA

Median [Q1 ; Q3]

Range

PTA air-bone gap

Median [Q1 ; Q3]

Range

+ information available for 127 ears. ${ }^{++}$information available for 32 ears. ${ }^{\S}$ Good function results were determined as a me

Table 2: Anatomical results

Characteristics

Number of ears

TM closed

TM satisfactory

Unsatisfactory results detail

Perforation

Myringitis

$\mathrm{OME}^{+}$

TM retraction

Cholesteatoma

Results are shown by Number (percentage). ${ }^{+}$Cases of isolated OME without associated TM retraction or cholesteatoma.

Table 3: Univariate analysis of risk factors for unsatisfactory results at 1-year follow-up

Risk factor

Number of ears

Age at surgery (years)

Age at surgery $<7$ years

Age at surgery $<8$ years

Age at surgery $<9$ years

Velopalatal cleft

Cranio-facial anomaly ${ }^{+}$

External auditory canal stenosis

History of confirmed allergy

History of contralateral tympanoplasty ${ }^{++}$

History of contralateral ventilation tube

Large perforation ${ }^{\S}$

Anterior or subtotal perforation

Perioperative inflammation ${ }^{\alpha}$

Junior surgeon

Results are shown by Number (percentage). Percentages of satisfactory and unsatisfactory results were calculated using the

\section{Fig 1. Patient inclusion flow-chart}

After the initial screening process and exclusion of non-eligible patients, 139 eardrums were analysed in the study. Patients who could not be contacted were excluded as it was not possible for them to be informed of the study (ethical limitation). The initial screening extracted all patients with perforations, thus patients with concurrent cholesteatoma or retraction pockets had to be removed secondarily.

Fig 2. Satisfactory results (closed without other complications) at one-year follow-up 
Representation of tympanic closure outcome at one-year follow-up according to age. Chi2 analysis found a risk factor of poor outcome for children operated on under the age of 8 years old $(p=0.02)$.

\section{FLOW CHART}
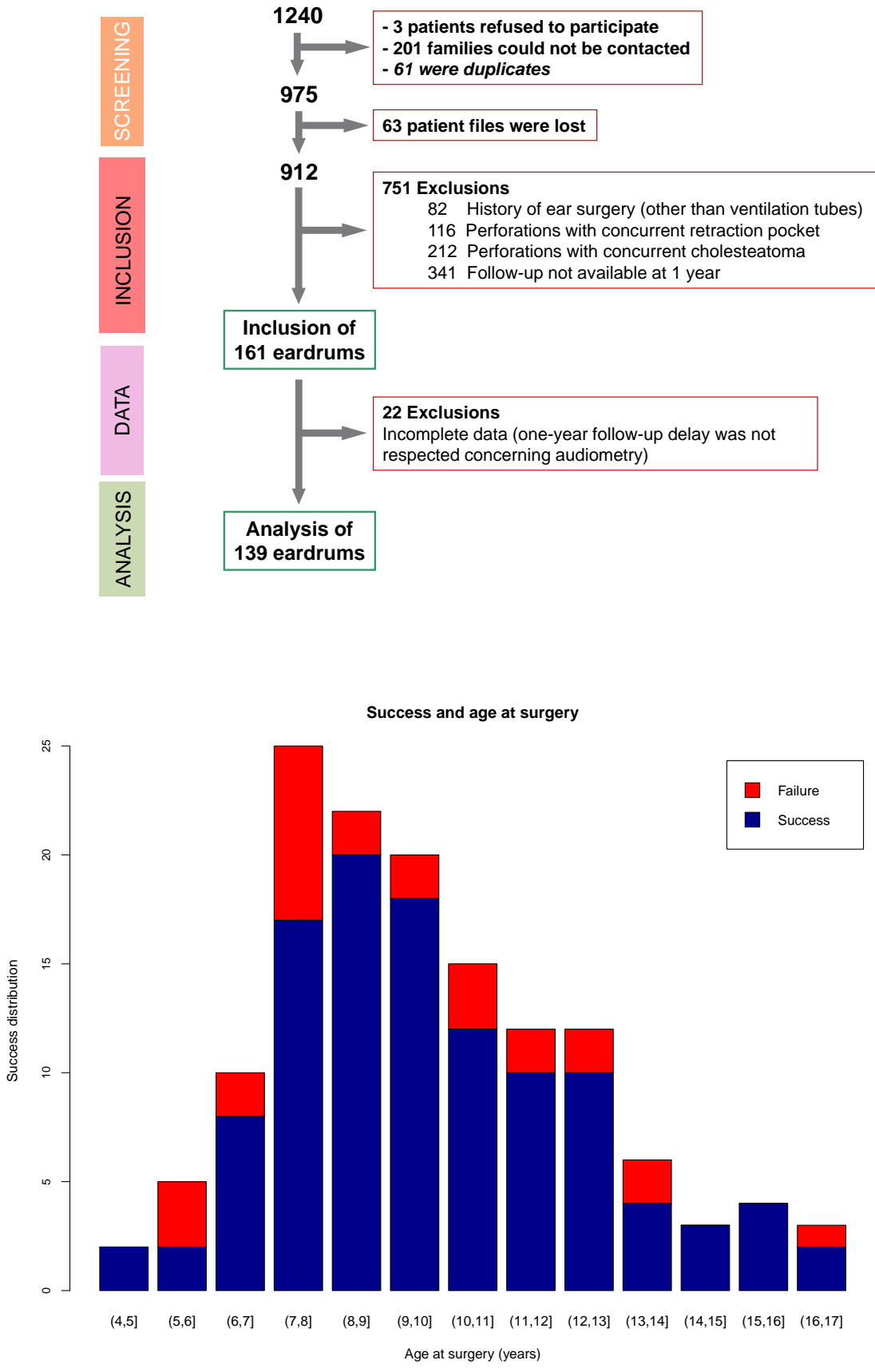